\title{
Proceeding
}

Supplementary Issue: Autumn Conferences of Sports Science. Costa Blanca Sports Science Events, 18-19 December 2020. Alicante, Spain.

\section{Wii Sport versus task-oriented training on gait in unilateral cerebral palsy: A randomized controlled trial}

\author{
NEHAD A. ABO-ZAID 14 , NESMA A. HELMY², NADER I. ELSAYED ${ }^{3}$, AMIRA H. MOHAMMED ${ }^{4}$ \\ ${ }^{1}$ Department of Physical Therapy for Pediatrics, Faculty of Physical Therapy, South Valley University, Qena, Egypt \\ 2Basic Science Department, Faculty of Physical Therapy, Beni Suef University, Egypt \\ ${ }^{3}$ Department of Physical Therapy for Orthopedics, Faculty of Physical Therapy, Beni Suef University, Egypt \\ ${ }^{4}$ Department of Physical Therapy for Pediatrics, Faculty of Physical Therapy, Delta University for Science and \\ Technology, Gamasa, Egypt
}

\begin{abstract}
Background: Unilateral cerebral palsy is a complex and heterogeneous disorder leading to a variety of functional impairment and gait disorders. Objective: To compare the effect of Wii Sport Training and Taskoriented Training on gait in children with unilateral cerebral palsy. Participants and Methodology: Sixty unilateral cerebral palsy children were selected in this study after the assessment of eligibility. Their ages ranged from eight to twelve years. They were randomly assigned into three groups of equal numbers. The control group (G1) received only a guideline protocol, group (G2) received the same guideline protocol plus Wii Sport Training and group (G3) received the same guideline protocol plus Task-oriented Training (TOT) group. All groups received the treatment protocol three times / weak for four successive months. Assessment of gait by 3D motion analysis (3DMA) system pre- and post-treatment that continued four months was conducted for all groups. Results: Post-treatment, significant improvement in the percent of weight support, stride length, step length, and cadence, in control, Wii Sport Training and TOT groups in favour of TOT group, also significant decrease in the percent of swing phase in all groups in favour of TOT group $(p<.05)$. Conclusions: Wii Sport Training and Task-oriented Training have a significant effect on gait in children with unilateral cerebral palsy in favour of Task-oriented Training group.

Keywords: Unilateral cerebral palsy; Gait; Wii Sport training; Task-oriented training.

\section{Cite this article as:}

Abo-Zaid, N.A., Helmy, N.A., Elsayed, N.I., \& Mohammed, A.H. (2021). Wii Sport versus task-oriented training on gait in unilateral cerebral palsy: A randomized controlled trial. Journal of Human Sport and Exercise, 16(2proc), S522-S531. doi:https://doi.org/10.14198/jhse.2021.16.Proc2.36

Corresponding author. Department of Physical Therapy for Pediatrics, Faculty of Physical Therapy, South Valley University, Qena, Egypt.

E-mail: dr.nehadahmed@svu.edu.eg

Abstract submitted to: Autumn Conferences of Sports Science. Costa Blanca Sports Science Events, 18-19 December 2020. Alicante, Spain.

JOURNAL OF HUMAN SPORT \& EXERCISE ISSN 1988-5202

(c) Faculty of Education. University of Alicante

doi:10.14198/jhse.2021.16.Proc2.36
\end{abstract}

S522 | 2021| Proc2 | VOLUME 16

C 2021 University of Alicante 


\section{INTRODUCTION}

Cerebral Palsy (CP) is a collection of permanent motor disorders of movement and posture resulting from brain damage before, during, or after birth that affects the motor system and leads to activity limitation (Miller \& Bachrach, 2017). The most common type of CP is unilateral CP that affecting one person per thousands of live births, characterized by a clinical pattern of unilateral motor impairment, movement disabilities, and postural disturbance (Robert \& Levin, 2018), that contributes to their gait abnormalities (Chen et al., 2013), these abnormalities resulting from functional strategies to compensate for the primary anomalies due to central nervous system damage (Szopa, Szopa \& Malgorzata, 2014).

In recent years, there has been increasing interest in the use of the Nintendo Wii as an active video games system that considered one of the most common virtual rehabilitation tools (VR). It is a technology-based game that provides people to expertise and move with computer-generated environments using their all senses, as touch, vision, and/or hearing (Brutsch, Schuler, Koenig, Zimmerli, Koeneke \& Lunenburger, 2010). It gives challenges, increasing curiosity, and promoting fantasy for children so they are deeply engaging with the intervention (Holden, Dyar \& Dayan, 2010). It includes the fundamental principles of the motor learning theories by enabling the user to unendingly see the movements in 3 dimensions from the screen, providing continuous purposeful and positive feedback leading to improve functional independence in daily tasks (Holden, 2005).

Task-oriented training (TOT) is a form of therapy with which patients 'practice context-specific motor tasks and receiving of feedback', it provides dynamic interactions between the individual, the task and the individual's environment, is considered a top-down approach that focused on each client's goal (Pendleton \& Schultz-Krohn, 2013). It can enhance motor function and improve muscle strength or performance (Byoung \& Yumi, 2013). With the development of TOT, the participants have shifted their interest from traditional inhibition, facilitation techniques, and minimizing deficits to more dynamic TOT that enhance functional improvement in all aspects of motor performance (Rensink, Schuurmans, Lindeman \& Hafsteinsdóttir, 2009).

Several studies were reported that Wii Sport Training and TOT have a great impact to improve motor function in children with gait problems. However, there are no studies that compare the effect of these two types of therapeutic interventions in clinical settings. So, our study aimed to compare the effect of Wii Sport Training as a form of VR and TOT on gait in children with unilateral CP. It was a hypothesis that there were no differences between the effects of Wii Sport Training and TOT on gait parameters in children with unilateral $\mathrm{CP}$.

\section{METHOD}

\section{Study design}

This randomized controlled study was conducted in the private outpatient clinic during the period from April 2017 to April 2019. Informed consent was submitted by the parents of each child. Our procedures had No: P.T.REC/012/002841 that approved by the Institutional Ethical Committee Clearance of the Faculty of Physical Therapy at Cairo University. The registration number of our study was: NCT04533789 that was registered on Clinicaltrial.gov.

\section{Participants}

Sixty unilateral CP children were selected from the private out-patient clinic of the Faculty of Physical Therapy, Delta University for Science and Technology and different Paediatrics Rehabilitation Centres, they 
were diagnosed as having unilateral CP based on careful clinical assessment by a physiotherapist. After screening, the selected children were from both sexes, their ages ranged from eight to twelve years, they were from both sexes, they had mild to moderate spasticity according to the Modified Ashworth Scale and between level I and II according to Growth Motor Function Classification System (GMFCS), they were stable medically and psychologically, they were able to follow verbal commands or instructions. We excluded from the study children who had any auditory or visual problems, children that had a history of epilepsy, children with fixed deformities in lower limb that interfere with walking, children with a history of any surgical interference in lower limbs for less than one year, children with unstable medical status especially those with cardiovascular disorders, mentally retardation, and un-cooperative children.

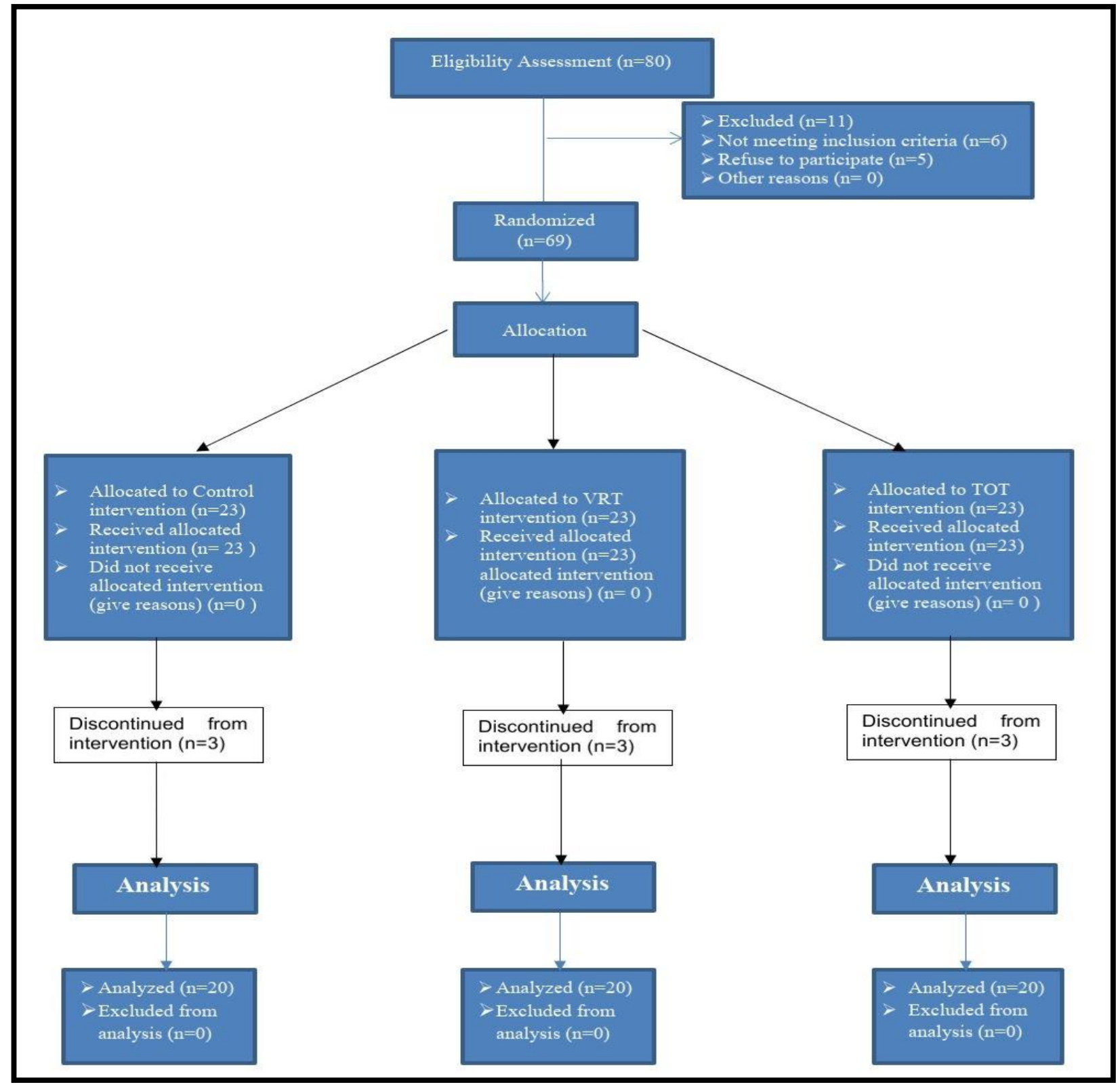

Figure 1. Flow chart. 


\section{Randomization}

Eighty children with unilateral CP were evaluated for eligibility; six children were excluded because they failed to fulfil the inclusion criteria and five parents refused to be enrolled in this study. Therefore, sixty-nine children with hemiplegic CP were included in this study, twenty-three children in each group. The selected children were divided randomly into three groups of equal numbers one control and two study groups; Wii Sport Training as a form of VR and TOT using random allocation software were used to minimize selection bias (Saghaei. 2004). For the control group, three children did not continue treatment program to the final assessment, while for the VR group; three children did not continue due to difficulties with Wii usage, while for the TOT group; three children did not continue due to traveling with parents to another country. So, for each group, twenty children were participated in a post-treatment assessment. A diagram of children's retention and randomization throughout the study is shown in Figure 1.

\section{Outcome measures}

3D motion analysis (3DMA), a video-based gait assessment system (CLIMA) was used to assess gait parameters including the percent of swing phase, percent of weight support, stride length, step length, and cadence. Gait evaluation was done by using a clinical 3DMA 6.11 software version. Cilma, STT Systems Company, Spain.

The calibration process of 3DMA was carried out by exposing the system to a known calibration tool named "calibration rod". This was a straight bar with markers evenly placed at fixed intervals. Before starting the calibration processes any extraneous reflections or spotlights were checked and removed. The calibration rod was put vertically during data collection. The capture area on the floor was delimited by using adhesive tapes.

The numbers of snapshots of the calibration rod in different positions all along the capture area were collected. The basic rules for a suitable positioning were as follow:

- All the rod markers could be seen by all the cameras.

- The calibration should not put twice in the same place.

All children were evaluated for percent of swing phase, percent of weight support, stride length, step length, and cadence using the 3DMA before and after four months of intervention.

\section{Intervention}

- Control group (G1): Children in this group received 60 minutes of a guideline protocol that was based on neurodevelopment basis that was included; standing on one foot, stride standing allowing weight shift from one limb to another, standing on balance board, stoop and recovery from standing position, balancing exercises from standing in the form of equilibrium, righting and protective reactions training, improving trunk control, balance training exercises, gait training exercises in an open environment and stretching exercises for elbow flexors and forearm supinator of upper limbs, hip flexors, and adductors, hamstrings and calf muscles of lower limbs (Saghaei, 2004), based on the neurodevelopmental basis for 60 min., 3 sessions/ week.

- VR group (G2): Children in this group received the same guideline protocol which was applied to the control group (G1) for 30 min in addition to virtual reality training using Wii device (Nintendo Wii)), for $30 \mathrm{~min}$ (Levitt, 2018).

- TOT group (G3): Children in this group received the same guideline protocol which was given to the control group (G1), VR group (G2) for 30 min. in addition to task-oriented training, for 30 min (Gatica, Méndez \& Guzman 2017). It included chairs with different heights, blocks with different heights, 
steppers with different heights, and stairs with different heights. All participants received different treatment protocol 3 sessions/ week for four successive months.

\section{Sample size}

To avoid type II error, a preliminary power analysis [F test, MANOVA: special effects and interaction, power $(1-a$ error $p)=.80, a=.05$, Pillai $V=0.21$, effect size $f 2(V)=0.117$, with a comparison of 3 independent groups, number of variables (mainly primary outcome $=5$, and number of predictors $=2$ ] determined a sample size of 57 for each group in the study using $\mathrm{G}^{*}$ power 3.1 software. The Pillai $V$ and the effect size were calculated according to a pilot study on 9 participants ( 3 in each group) considering swing time, support time as a primary outcome.

\section{Statistical analysis}

SPSS Package program version 25 for Windows (SPSS, Inc., Chicago, IL), data was screened before the final analysis for normality assumption test by using the Shapiro-Wilk test $(p>.05)$. One way analysis of variance (ANOVA test) was used to compare three groups for all demographic variables, also the Chi-square test was used to compare between 3 groups for gender variable.

Multivariate tests by MANOVA for outcome measures (Table 2) was conducted to compare gait parameters (\% of swing phase, $\%$ of support phase, step length, stride length, and cadence among three groups (control G, VR G and TOT G), followed by post hoc tukey test in case of significant result for compare between each two independent group to explore previously where the significant difference occurs. P-value was used to indicate the level of significance $(p<.05)$.

\section{RESULTS}

A total of 60 patients participated in this study; they were distributed randomly into 3 groups of equal numbers. Before treatment, there were no significant differences in age, weight, height, BMI, and gender between the groups (Table 1). Also, there was a statistically significant $(p<.05)$ effects due to main effects of tested groups, measuring time and group $\mathrm{x}$ time interaction (Table 2).

Table 1. Comparison means values of demographic data between groups.

\begin{tabular}{|c|c|c|c|c|c|c|}
\hline \multirow[b]{2}{*}{ Variables } & \multicolumn{3}{|c|}{ Mean \pm Standard deviation } & \multirow{2}{*}{ F-value } & \multirow[b]{2}{*}{$p$-value } & \multirow[b]{2}{*}{ Significance } \\
\hline & G1 & G2 & G3 & & & \\
\hline Age & $10.3 \pm 1.17$ & $9.9 \pm 0.91$ & $10.15 \pm 1.09$ & 211 & .4906 & \\
\hline & $33.75 \pm 4.6$ & \pm 3.6 & 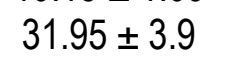 & 1.4 & .24 & \\
\hline Height $(\mathrm{cm})$ & $127.3 \pm 8.5$ & $123.6 \pm 5.26$ & $123.8 \pm 7.7$ & 1.632 & .2045 & \\
\hline BMI (kg/m²) & $20.73 \pm 0.48$ & $20.75 \pm 0.92$ & $20.8 \pm 0.82$ & 0.03451 & .9661 & cant \\
\hline $\begin{array}{l}\text { Gender } \\
\text { (Male: Female) }\end{array}$ & $\begin{array}{c}14(70 \%): \\
6(30 \%)\end{array}$ & $\begin{array}{c}13(65 \%): \\
7(35 \%)\end{array}$ & $\begin{array}{c}16(80 \%): \\
4(20 \%)\end{array}$ & & .5630 & \\
\hline
\end{tabular}
Note: $p$-value: probability value. ${ }^{*}$ Significant ( $p$-value <.05).

Table 2. Main effects of independent variables by MANOVA test for all dependent measuring variables.

\begin{tabular}{lcccc}
\hline Source of variation & Wilk's Lambada Value & Partial Eta $^{2}(\mathbf{\eta} 2)$ & F-value & p-value \\
\hline Tested groups effect & 0.208 & 0.544 & 7.451 & $.0001^{*}$ \\
Measuring period effect & 0.003 & 0.977 & 461.060 & $.0001^{*}$ \\
Interaction effect & 0.023 & 0.745 & 69.551 & $.010^{*}$ \\
\hline
\end{tabular}
Note: $p$-value: probability value. ${ }^{*}$ Significant ( $p$-value <.05). 
Comparison within all groups between pre- and post-treatment mean values showed, there was a significant decrease in post-treatment in percent of swing phase and significantly increase in post-treatment in percent of support phase, step length, stride length, and cadence $(p<.05)$, (Table 3$)$.

Table 3. The design MANOVA for all dependent measuring variables within and between groups.

\begin{tabular}{|c|c|c|c|c|c|c|}
\hline Variables & & $\begin{array}{l}\text { Control } \\
\text { group }(n=20)\end{array}$ & $\begin{array}{l}\text { VR } \\
\text { group }(n=20)\end{array}$ & $\begin{array}{l}\text { TOT } \\
\text { group }(n=20)\end{array}$ & & \\
\hline \multirow{5}{*}{ Swing \% } & Pre-treatment & $47.38 \pm 2.2$ & $47.08 \pm 1.99$ & $46.82 \pm 2.6$ & 0.298 & 0.7437 \\
\hline & Post-treatment & $45.87 \pm 1.5$ & $44.92 \pm 1.29$ & $42.89 \pm 0.77$ & 31.153 & 0.0001 \\
\hline & Improvement \% & $3.18 \%$ & $4.85 \%$ & $8.39 \%$ & & \\
\hline & F-value & 2.1919 & 16.6975 & 15.4457 & & \\
\hline & $p$-value & .0103 & .0003 & .0001 & & \\
\hline \multirow{5}{*}{ Support \% } & Pre-treatment & $52.62 \pm 2.2$ & $52.92 \pm 1.99$ & $53.18 \pm 2.6$ & 0.298 & 0.7437 \\
\hline & Post-treatment & $54.13 \pm 1.5$ & $55.08 \pm 1.3$ & $57.12 \pm 0.77$ & 31.153 & 0.0001 \\
\hline & Improvement \% & $2.86 \%$ & $4.08 \%$ & $7.40 \%$ & & \\
\hline & F-value & 6.3017 & 16.6975 & 11.3817 & & \\
\hline & $p$-value & .0103 & .0003 & .0001 & & \\
\hline \multirow{5}{*}{ Stride Length } & Pre-treatment & $0.63 \pm 0.05$ & $0.61 \pm 0.05$ & $0.61 \pm 0.03$ & 1.306 & 0.2866 \\
\hline & Post-treatment & $0.65 \pm 0.04$ & $0.69 \pm 0.036$ & $0.72 \pm 0.035$ & 15.204 & 0.0002 \\
\hline & Improvement \% & $3.17 \%$ & $13.11 \%$ & $18.03 \%$ & & \\
\hline & F-value & 2.2821 & 2.2298 & 0.7134 & & \\
\hline & $p$-value & .0078 & .0001 & .0001 & & \\
\hline \multirow{5}{*}{ Step Length } & Pre-treatment & $0.314 \pm 0.02$ & $0.306 \pm 0.027$ & $0.308 \pm 0.016$ & 0.643 & 0.4932 \\
\hline & Post-treatment & $0.32 \pm 0.02$ & $0.34 \pm 0.018$ & $0.358 \pm 0.018$ & 15.654 & 0.0001 \\
\hline & Improvement \% & $1.91 \%$ & $11.11 \%$ & $16.23 \%$ & & \\
\hline & F-value & 1.8389 & 2.2298 & 0.8830 & & \\
\hline & $p$-value & .2187 & .0001 & .0001 & & \\
\hline \multirow{5}{*}{ Cadence } & Pre-treatment & $82.72 \pm 4.874$ & $83.91 \pm 3.621$ & $84.79 \pm 1.374$ & 1.664 & 0.2253 \\
\hline & Post-treatment & $84.4 \pm 3.965$ & $87.13 \pm 3.025$ & $91.15 \pm 3.94$ & 17.106 & 0.0001 \\
\hline & Improvement \% & $2.03 \%$ & $3.83 \%$ & $7.50 \%$ & & \\
\hline & F-value & 1.4288 & 9.2705 & 0.1213 & & \\
\hline & $p$-value & .0231 & .0009 & .0001 & & \\
\hline
\end{tabular}

Table 4. Post Hoc Tukey test for comparison of the mean values of different variables of gait parameters indices post-treatment between each pair of the three groups.

\begin{tabular}{lcccccc}
\hline \multirow{2}{*}{ Variables } & \multicolumn{5}{c}{ Post treatment } \\
\cline { 2 - 7 } & \multicolumn{2}{c}{ Control vs. VR } & \multicolumn{2}{c}{ Control vs. TOT } & \multicolumn{2}{c}{ VR vs. TOT } \\
\cline { 2 - 7 } & $\begin{array}{c}\text { Mean } \\
\text { difference }\end{array}$ & $p$-value & $\begin{array}{c}\text { Mean } \\
\text { difference }\end{array}$ & $p$-value & $\begin{array}{c}\text { Mean } \\
\text { difference }\end{array}$ & $p$-value \\
\hline Swing \% & 0.9510 & $.0443^{*}$ & 2.989 & $<.0001^{*}$ & 2.038 & $<.0001^{*}$ \\
Support \% & -0.9510 & $.0443^{*}$ & -2.989 & $<.0001^{*}$ & -2.038 & $<.0001^{*}$ \\
Stride Length & -0.0320 & $.0455^{*}$ & -0.0650 & $.0011^{*}$ & -0.0330 & $.0250^{*}$ \\
Step Length & -0.01875 & $.0257^{*}$ & -0.03350 & $.0010^{*}$ & -0.01475 & $.0467^{*}$ \\
Cadence & -2.724 & $.0357^{*}$ & -6.748 & $<.0001^{*}$ & -4.024 & $.0059^{*}$ \\
\hline
\end{tabular}


Comparison between all groups between pre-treatment mean values showed, there was no significant difference between all three groups, but post-treatment there was a significant decrease in post-treatment in percent of swing phase and significantly increase in post-treatment in percent of support phase, step length, stride length, and cadence $(p<.05)$, (Table 3). Post-treatment Post hoc Tukey test revealed that there was a significant difference between control group and VR group in favour to VR group, there was a significant difference between control group and VR group in favour to VR group, and there was a significant difference between VR group and TOT group in favour to TOT group, (Table 4) Comparison between three groups reveal there was a significant improvement in favour to TOT group.

\section{DISCUSSION}

Virtual Reality Training and Task-Oriented Training are new and modern treatment strategies, several studies showed that it had a good impact and proven to be useful adjunct techniques to the traditional methods for rehabilitation of unilateral cerebral palsied children. So, our study aimed to compare the effect of Wii Sport Training as a form of VR and TOT in improving gait parameters in children with unilateral CP.

Children with unilateral CP suffer from lower limb asymmetrical behaviour that is clinically manifested as gait asymmetry (Han \& Chung, 2016). They have poor stability resulting from fluctuations of the COG (Patterson, Gage, Brooks, Black \& Mcllroy, 2010). The reduction in velocity, cadence, and stride length are common gait dysfunctions in children with unilateral CP (Wang, 2012; Armand, Decoulon \& Bonnefoy-Mazure, 2016). The age of our participant was ranged from 8 to 12 years of old, as feed-forward control of goal-directed locomotion appears in mid-childhood age in gait development and becomes increasingly important afterward (Marshall, Teasell, Bayona, Lippert, Chundamala \& Villamere, 2007). Also, they are able to develop lower segment movements to overcome obstacles of moderate height in the manner resemble adults (Grasso, Assaiante, Prevost \& Berthoz, 1998).

The pre-treatment means values showed no significant differences between three groups in gait parameters including percentage (\%) of swing phase, percentage (\%) of weight support, stride length, step length and cadence $(p>.05)$. Comparing the post-treatment to the pre-treatment mean values showed improvement in gait parameters including percentage $(\%)$ of swing phase, percentage $(\%)$ of weight support, stride length, step length, and cadence in children of the three groups $(p<.05)$, while this improvement was in favour of TOT group. These significant improvements in all groups, this may be attributed to neuro-development training that was used resulting in modulation of muscle tone and improving postural alignment, this supported by Ottenbacher, Biocca, DeCremer, Gevelinger, Jedlovec \& Johnson, (1986) who concluded that the guideline protocol (based on neurodevelopment basis) has a direct effect on facilitation of postural control and development of lower extremities and trunk normal movement patterns varieties (McFadyen, Malouin \& Dumas, 2001). Also, the rehabilitation programs based on neuro-developmental basis provide increasing general physical capacity and functional independence for children with CP (Ottenbacher, Biocca, DeCremer, Gevelinger, Jedlovec \& Johnson, 1986).

Also, Dewar, Love \& Johnston, (2015) reported that repetition is the basis of neuroplasticity, so repetition of a motor task is fundamental in improving motor control during rehabilitation. This improvement may be attributed to that VR training can facilitate motor rehabilitation through several underlying mechanisms such as amplification of sensorimotor representation by augmented sensory feedback, reduction of cognitive load, highlight any error through feedback (Dewar, Love \& Johnston, 2015), reduction of musculoskeletal coactivation (White, Burdick, Fulk, Searleman \& Carroll, 2005), increased arousal and motivation (Yoo, Lee, Sim, You \& Kim, 2014). Virtual reality acts to active the primary sensorimotor cortex on the same side of the 
lesion in hemiplegic CP children which may be related to increase in their functional abilities (Epure \& Holte, 2018). This activation is obtained through performance of similar tasks by other persons in the manner shaped by VR environments (You, Jang, Kim, Kwon, Barrow \& Hallett, 2005).

Regarding the results of the TOT group, the results of this study may be due to the selected interventions which were matched with the daily activities aiming to maximize the transfer to normal routine and to achieve gains in the functional aspect of mobility (Magill, 1998). As a training principle, there was a direct relation between the trained function and the force and power of the acting muscles (Buchner, Larson \& Wagner, 1996). The TOT set the involvement of the training in everyday activities as a principle to achieve motor control. Based on this principle, we could attribute the improvement in the measured variables of TOT group specially stride length and cadence. It improve the stability for hip and knee joints which directly improve the stability in stance phase so the children walk with longer stride length and push off actively their ankles (Salbach, Mayo, Wood-Dauphinee, Hanley \& Richards, 2004; Pang, 2014).

\section{CONCLUSION}

The finding of our study showed that TOT has a positive impact on gait than Wii Sport Training as a form of VR in children with unilateral $C P$.

\section{Limitations}

No time for follow up may limit the maintenance of the statistical results to predict the loss or relapse in all gait parameters. So, further studies with prolonged time and follow-up after treatment program are needed to confirm our findings.

\section{Conflict of interest}

Authors have not declared any conflict of interest.

\section{Role of funding source}

No benefits or funds were received in support of this study.

\section{REFERENCES}

Armand S., Decoulon G. and Bonnefoy-Mazure A. (2016): Gait analysis in children with cerebral palsy. EFORT Open Rev; 1:448-60. https://doi.org/10.1302/2058-5241.1.000052

Brutsch K., Schuler T., Koenig A., Zimmerli L., Koeneke S.M. and Lunenburger L. (2010): Influence of virtual reality soccer game on walking performance in robotic assisted gait training for children. Journal of neuroengineering and rehabilitation; 7:15. https://doi.org/10.1186/1743-0003-7-15

Buchner D.M., Larson E.B. and Wagner E.H. (1996): Evidence for a nonlinear relationship between leg and gait speed. Age Ageing; 25:386-91. https://doi.org/10.1093/ageing/25.5.386

Byoung H. and Yumi K. (2013): Clinical Usefulness of Child-centered Task-oriented Training on Balance Ability in Cerebral palsy. J PhysTherSci; 25(8): 947-51. https://doi.org/10.1589/jpts.25.947

Chen L., Chen Y., Liaw Y., Chung Y., Wang J. and Hong H. (2013): Efficacy of home-based virtual cycling training on bone mineral density in ambulatory children with cerebral palsy. Osteoporosis Int; 24:1399-406. https://doi.org/10.1007/s00198-012-2137-0

Dewar R., Love S. and Johnston L.M. (2015): Exercise interventions improve postural control in children with cerebral palsy: a systematic review. Dev Med Child Neurol; 57:504-20. https://doi.org/10.1111/dmcn.12660 
Epure P. and Holte M.B. (2018): Analysis of motivation in virtual reality stroke rehabilitation. In: Interactivity, Game Creation, Design, Learning, and Innovation. Cham: Springer International Publishing. p. 282. https://doi.org/10.1007/978-3-319-76908-0 27

Gatica R.V. and Méndez R.G. (2014): Virtual reality interface devices in the reorganization of neural networks in the brain of patients with neurological diseases. Neural Regen Res; 9:888-96. https://doi.org/10.4103/1673-5374.131612

Gatica R.V., Méndez R.G. and Guzman M.E. (2017): Does Nintendo Wii Balance Board improves standing balance? A randomized controlled trial in children with cerebral palsy. Eur J PhysRehabil Med; 53(4):535-544.

Ghai S. and Ghai I. (2019): Virtual Reality Enhances Gait in Cerebral Palsy: A Training Dose-Response Meta-Analysis. Front. Neurol; 10:236. https://doi.org/10.3389/fneur.2019.00236

Grasso R., Assaiante C., Prevost P. and Berthoz A. (1998): Development of anticipatory orienting strategies during locomotor tasks in children. NeurosciBiobehav Rev; 22(4):533-9. https://doi.org/10.1016/S0149-7634(97)00041-9

Han H.K. and Chung Y.J. (2016): Effects of task-oriented training for gross motor function measure, balance and gait function in persons with cerebral palsy. PhysTherRehabilSci; 5:9-14. https://doi.org/10.14474/ptrs.2016.5.1.9

Holden M.K. (2005): Virtual environments for motor rehabilitation: review. Cyberpsychology \& behavior: the impact of the Internet, multimedia and virtual reality on behavior and society; 8 (3):187-211; discussion 2-9. https://doi.org/10.1089/cpb.2005.8.187

Holden M.K., Dyar T.A. and Dayan L. (2010): Cimadoro, Telerehabilitation using a virtual environment improves upper extremity function in patients with stroke, IEEE Transactions on Neural Systems.

Levitt S. (2018): Treatment of cerebral palsy and motor delay, 6th Ed., Blackwell Publishing, Oxford; 157175. https://doi.org/10.1002/9781119373902

Magill R.A. (1998): Motor learning. Concepts and applications, fifth ed. Boston: McGraw Hill.

Marshall S., Teasell R., Bayona N., Lippert C., Chundamala J. and Villamere J. (2007): Motor impairment rehabilitation post acquired brain injury. Brain Inj; 21:133-60. https://doi.org/10.1080/02699050701201383

McFadyen B.J., Malouin F. and Dumas F. (2001): Anticipatory locomotor control for obstacle avoidance in mid-childhood aged children. Gait Posture; 13(1):7-16. https://doi.org/10.1016/S09666362(00)00090-4

Miller F. and Bachrach S. (2017): Cerebral Palsy: A complete guide for cargiving. 3rd edition, Part one: What is cerebral palsy. Publisher: John Hopking University Press, Baltimore; 3-28.

Ottenbacher K., Biocca Z., DeCremer G., Gevelinger M.,Jedlovec K. and Johnson M. (1986): Quantitative Analysis of the Effectiveness of Pediatric Therapy Emphasis on the Neurodevelopmental Treatment Approach. Physical therapy; 66: 1095-1101. https://doi.org/10.1093/pti/66.7.1095

Pang M.Y. (2014): Task-specific and impairment-based training improve walking ability in stroke. J Physiother; 60: 110. https://doi.org/10.1016/j.jphys.2014.05.011

Patterson K.K., Gage W.H., Brooks D., Black S.E. and Mcllroy W.E. (2010): Evaluation of gait symmetry after stroke: a comparison of current methods and recommendations for standardization. Gait Posture; $31: 241-6$. https://doi.org/10.1016/j.gaitpost.2009.10.014

Pendleton H. and Schultz-Krohn W. (2013): Pedretti's occupational therapy: Practice skills for physical dysfunction. St. Louis, Missouri: Elsevier.

Rensink M., Schuurmans M., Lindeman E. and Hafsteinsdóttir T. (2009): Task oriented training in rehabilitation after stroke: systematic review. J AdvNurs.65(4):737-54. https://doi.org/10.1111/j.1365$\underline{2648.2008 .04925 . x}$ 
Robert M.T. and Levin MF. (2018): Validation of reaching in a virtual environment in typically developing children and children with mild unilateral cerebral palsy. Dev Med Child Neurol; Feb 10:159-182. https://doi.org/10.1111/dmcn.13688

Saghaei M. (2004): Random allocation software for parallel-group randomized trials. BMC Med Res Methodol; 4:26. https://doi.org/10.1186/1471-2288-4-26

Salbach N.M., Mayo N.E., Wood-Dauphinee S., Hanley J.A. and Richards C.L. (2004): A task-orientated intervention enhances walking distance and speed in the first year post stroke: a randomized controlled trial. ClinRehabil; 18: 509-519. https://doi.org/10.1191/0269215504cr763oa

Szopa A., Szopa D. and Malgorzata C.A. (2014): Gait pattern differences in children with unilateral cerebral palsy. Research in Developmental Disabilities; $35 . \quad 2261-2266$. https://doi.org/10.1016/j.ridd.2014.05.020

Wang X. (2012): Gait analysis of children with spastic hemiplegic cerebral palsy. Neural Reg Res; 7:1578-84.

White D., Burdick K., Fulk G., Searleman J., and Carroll J. (2005): A virtual reality application for stroke patient rehabilitation. In: IEEE International Conference Mechatronics and Automation. Singapore; 1081-1086. https://doi.org/10.1109/ICMA.2005.1626702

Yoo J.W., Lee D.R., Sim Y.J., You J.H. and Kim C.J. (2014): Effects of innovative virtual reality game and EMG biofeedback on neuromotor control in cerebral palsy. Biomed Mater Eng; 3613-8. https://doi.org/10.3233/BME-141188

You S.H., Jang S.H., Kim Y.H., Kwon Y.H., Barrow I. and Hallett M. (2005): Cortical reorganization induced by virtual reality therapy in a child with hemiparetic cerebral palsy. Dev Med Child Neurol; 47:628-35. https://doi.org/10.1017/S0012162205001234

\section{(@) $(\Theta \Theta \Theta$}

This work is licensed under a Attribution-NonCommercial-NoDerivatives 4.0 International (CC BY-NC-ND 4.0). 